\title{
Melatonin attenuates vascular calcification by activating mitochondrial fusion and mitophagy via an AMPK/OPA1 signaling pathway
}

chenweiren chen ( $\nabla$ chen_weiren@sina.com )

pla general hospital

jia qi yang

anzhen hospital

fang liu

anzhen hospital

xue qin shen

anzhen hospital

yuan sha

anzhen hospital

Research

Keywords: Melatonin, vascular calcification, mitochondrial fusion,mitophagy, AMPK, OPA1

Posted Date: March 23rd, 2020

DOI: https://doi.org/10.21203/rs.2.17699/v2

License: (c) (1) This work is licensed under a Creative Commons Attribution 4.0 International License.

Read Full License 
The authors have withdrawn this preprint from Research Square 